\title{
Retro-reflective Characteristics of Transparent Screen for Head Mounted Projection Displays
}

\author{
Shoaib R Soomro*, Hakan Urey \\ Electrical Engineering Department and Optical Microsystems Laboratory, Koc University, Istanbul, Turkey \\ *Corresponding author: ssoomro13@ku.edu.tr
}

\begin{abstract}
Retro-reflective features of microbeads based transparent screen are investigated. Analytical expression of reflection cone is formulated and experimentally validated. Screen luminance for different viewing conditions is calculated when used with HMPD.
\end{abstract}

OCIS codes: (120.2040) Displays, (120.2820) Heads-up displays, (220.0220) Optical design and fabrication

\section{Introduction}

Transparent screens find great potential in augmented reality applications. Transparent displays can be fabricated using transparent liquid crystals and light emitting diodes [1], however such displays are not scalable and have limited transparency. Several projection based transparent screens are also demonstrated using micro-lens array [2], resonant nano-particles [3] and holographic elements [4], but such screens suffer from lower optical gain and transparency, are hard to fabricate in large format, and are limited to single color due to wavelength selective operation.

Here we explore the characteristic of transparent retro-reflective screens for head mounted projection displays (HMPD). The transparent screen is based on retro-reflective microbeads partially patterned on see-through surface. We present an analytical expression to classify the profile of reflective cone (eye box) of screen. The analytical expression is validated by experimentally measuring the retro-reflective coefficient of the screen as a function of viewing angle. Luminance of transparent screen paired with a HMPD is calculated under different viewing conditions.

\section{Transparent Retro-reflective Screen}

A diffused screen scatters light equally in a hemispherical volume, while a microbeads based retro-reflective surface directs light towards source in the shape of angular cone. Due to shorter eye-projector distance in HMPDs, the retroreflective screen can provide higher gain using low power mobile projectors. Optimizing the screen transparency and gain, a transparent retro-reflective screen can be realized by partially depositing retro-reflective microbeads on optically clear substrate. To achieve uniform surface coverage, the retro-reflective material can be dispersed in the shape of tiny structures irresolvable by bare eye. For a working distance of $>60 \mathrm{~cm}$, the feature size of $200 \mathrm{um}$ is sufficient to achieve smooth and transparent screen.

We fabricated a transparent retro-reflective screen using above mentioned technique. We used hemi-spherically Alcoated microbeads with a refractive index of 1.9 and a mean diameter of $40 \mathrm{um}$. Optically clear acrylic sheet was used as substrate material. The microbeads were bonded to the substrate in square shaped micro islands of $200 \times 200 \mathrm{um}^{2}$ providing $25 \%$ surface filling, while $75 \%$ of surface area was left see-through.

\section{Retro-reflective Coefficient of Transparent Screen}

Our transparent retro-reflective screen reflects incident light in the form of angular cone. The microbeads give a Gaussian shaped angular cone, while the size of microbeads introduces diffraction in the reflected cone. Combining both, the reflection cone of transparent retro-reflective screen can be expressed as;

$$
R_{c}(\alpha)=\left(\frac{I_{p}}{I_{0}} e^{\frac{-\alpha^{2}}{2 \sigma^{2}}}+\frac{I_{b}}{I_{0}}\right)\left(\left(\operatorname{sinc} \frac{\alpha}{W}\right)^{2}+\frac{I_{d}}{I_{0}}\right)
$$

Where $R_{c}$ is coefficient of retro-reflectivity as function of viewing angle $\alpha, I_{0}$ source intensity, $I_{p}$ is captured intensity at reflected peak and $I_{b}$ is the bias intensity, both $I_{p}$ and $I_{b}$ depends on refractive index of microbeads. $\sigma$ is the constant for aperture of light source, $W$ represents width of the diffraction spot and is dependent on the size of microbeads and $I_{d}$ is amount of diffused intensity due to surface imperfection. The eye-projector distance (e) and viewer-to-screen distance (d) define the viewing angle $(\alpha)$ to screen as: $\alpha=\tan ^{-1}(e / d)$. With decreasing eyeprojector distance and increasing viewer-to-screen distance, the viewing angle decreases and viewer moves toward retro-reflective cone. The luminance of a diffused screen, illuminated with a projector of $P_{l}$ lumens across projection area $A=k \cdot d^{2}$ can be expressed as $L_{d}=P_{l} /(\pi A)$. Using eq.(1) and by dividing viewing space into small segments of solid angles $(\psi)$, the luminance formula can be modified for the transparent retro-reflective screen as; 


$$
L_{r}=R(\alpha) \frac{P_{l}}{\psi A}
$$

Eq. (2) shows that luminance of the transparent retro-reflective screen for changing viewer-to-screen distance can be calculated by knowing the eye-projector distance and reflection cone profile of screen.

\section{Experimental Results and Discussion}

To validate the analytical expression of reflected cone and calculate the luminance of transparent screen, the retroreflective coefficient of transparent screen was measured with a photometric setup as shown in fig.1(a). The screen sample was illuminated by a $632 \mathrm{~nm}$ collimated $\mathrm{HeNe}$ laser. The amount of reflected power as a function of viewing angle was measured with a detector. Fig.1(b) shows measured retro-reflective coefficient and its best analytical fit. The analytical model closely fits with experimental measurements. The screen provides an angular cone of about $2^{\circ}$, which is closer to the diffraction spot width of microbeads used in the screen.
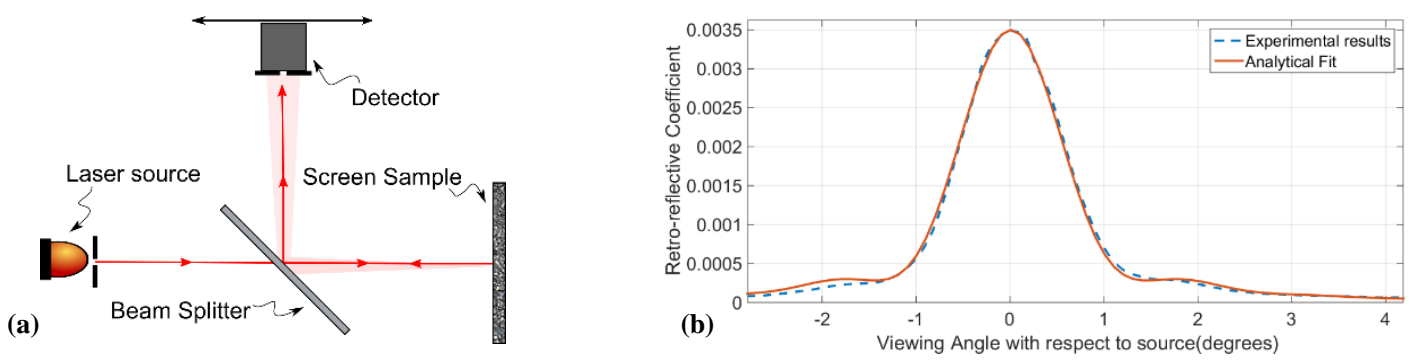

Fgure-1: (a) Illustration of experimental setup used to take measurements and (b) measurement results and comparison with analytical formulation.

Assuming a 15 lumen mobile projector, the luminance of transparent screen as function of viewer-to-screen distance is calculated using eq. (2), where $R_{c}$ is replaced with experimental measurements. As shown in fig.2(a), the screen provides exceptional luminance, when eye-projector distance is minimized and high optical gain (compared to diffused screen) at larger working distance. Each luminance curve shows the valley due to diffraction minima in reflection cone (fig.1(b)). The luminance valley can be compensated by using variable size microbeads.
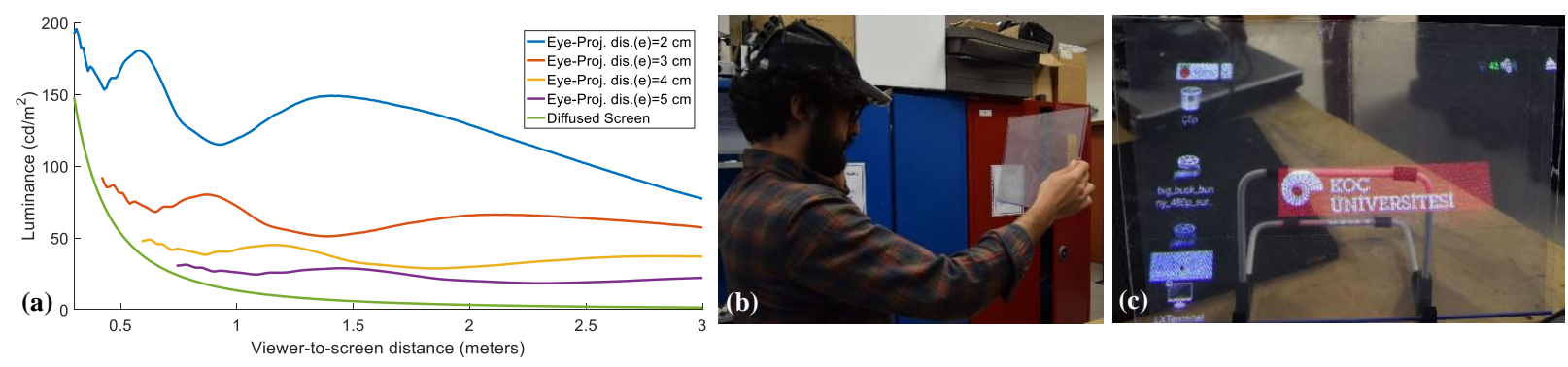

Figure-2: (a) Luminance of screen for different eye-projector distances, (b) Viewer using transparent screen, and (c) content as seen by viewer

Fig.2(b) shows a viewer using transparent retro-reflective screen with a laser pico-projector (Microvision) based HMPD. Fig.2(c) shows the bright floating content displayed on transparent screen as seen by viewer.

\section{Conclusion}

We investigated a transparent retro-reflective screen by formulating the analytical expression for its retro-reflective cone and compared it to experimentally measured retro-reflective coefficient. The luminance of screen as function of screen-to-viewer distance is also measured by using experimental results. The performance of screen can be further improved by changing the surface filling and widening retro-reflective cone using small sized microbeads.

This research is sponsored by European Research Council Advanced Grant (ERC-AdG) Wear3D Project No: 340200.

\section{References}

[1] Görrn, Patrick, et al. "Towards see-through displays: fully transparent thin-film transistors driving transparent organic light-emitting diodes." Advanced Materials 18 (2006): 738-741.

[2] Hedili, M. Kivanc, Mark O. Freeman, and Hakan Urey. "Transmission characteristics of a bidirectional transparent screen based on reflective microlenses." Optics express 21 (2013): 24636-24646.

[3] Hsu, Chia Wei, et al. "Transparent displays enabled by resonant nanoparticle scattering." Nature communications 5 (2014).

[4] Hong, Keehoon, et al. "Two-dimensional and three-dimensional transparent screens based on lens-array holographic optical elements." Optics express 22 (2014): 14363-14374. 